\title{
Structural Health Monitoring by Acoustic Emission Technique
}

\author{
Md. Tawhidul Islam Khan \\ Additional information is available at the end of the chapter \\ http://dx.doi.org/10.5772/intechopen.79483
}

\begin{abstract}
Elastic wave, which is formed due to sudden rearrangement of stresses in a material, is called acoustic emission (AE). It is widely used in nondestructive testing (NDT) of materials and structures especially in health monitoring of structures for damage detection. When a body is subjected to an external force (in the form of changing pressure, load, or temperature), any micro fracture inside the body releases energy in the form of AE wave, which is received by sensor and later on is converted to electrical signal for inspection. In early stage, major importance was given on studying the $\mathrm{AE}$ characteristics during the deformation and fracture on various materials (by J. Kaiser in Germany in 1950 and B. H. Schofield in the USA in 1954). Nowadays, lots of research are conducting on formulating the theories behind $\mathrm{AE}$ formation, propagation, and inspection in various fields as an important health monitoring tool for NDT. In this chapter, I would like to elaborate a "feature outlook of AE" based on past, present, and future perspectives; "AE monitoring" procedure based on theoretical and experimental perspectives; and smart applications in structural health monitoring based on industrial and biostructural perspectives with related figures and tables.
\end{abstract}

Keywords: structural health monitoring, nondestructive testing, acoustic emission technique, industrial applications, biomedical engineering application

\section{Introduction}

Structural health monitoring (SHM) refers to the theme of damage detection, evaluation, and characterization strategy of an engineering structure through time ranging feature extraction by sensors. Analytical and statistical representations, periodic forms in most cases, of damage-sensitive features of the structure focus on the monitoring system about the present status of structural health condition. 


\subsection{Acoustic emission (AE) in SHM}

Many conventional techniques are proposed by many engineers and scientists for health monitoring of structures, and maximum of them is nondestructive testing (NDT) type methods. Again, in many NDT systems, testing loadings are applied before or after the testing and are widely used as an active method, where signals or energy is delivered from the outside to the testing body. Contrary to active NDT, acoustic emission is widely used as passive NDT method in structural health monitoring, where external energy is not needed to supply the testing structure. The stimulated internal energy of the structure is received in this acoustic emission technique as health monitoring features. Due to this unique characteristic, acoustic emission technique has become very simple; however, accuracy or acquisition sensitivity is very high. Therefore, acoustic emission technique is becoming popular day by day in all types of structural monitoring fields [1-4].

A dominating attribute of acoustic emission technique is its application ability in its loading condition. Therefore, it provides instant damage information within a short period of time. Thus, acoustic emission monitoring tests are often performed in the operating conditions of the structure. As a result, adequate damage information even in minute state triggers the acoustic emission technique as a valuable health monitoring method. Dynamic characterization of any structural damage has become advantageous in this unique monitoring system. Furthermore, since acoustic emission technique is applied in dynamic feature, early stage of any damage can be characterized in this technique. Therefore, easy and adequate measures against any matured fracture or damage can be adopted in the presented fault detection technique [5-8].

Among many important features of acoustic emission technique, source location ability of involved damage inside the material attracts people in application of this technique as well. Following the traveling information of AE hits and applying to preferable algorithms, source location of AE event is performed. Based on the availability of sensors, dimension of source location is defined. For example, if two AE sensors are available, a single degree of source location is applicable; if three sensors are available, two-dimensional source location is applicable; if four or more sensors are available, three-dimensional source location is applicable. Since signal velocity influences the source location very much, velocity modes greatly affect the source location technique as well [9-12].

Present acoustic emission technique can quantify qualitative measure of the defects of a structure. For getting quantitative information of the damage, it often prefers to get supports of ultrasonic testing method. However, ultrasonic technique is necessary to apply in static condition, which is contradictory to the basic principle of acoustic emission. Therefore, pre- or postquantitative diagnosis is suitable for getting damage sizing inside the structure [13].

In addition to many advantages of AE applications in nondestructive testing environment, entire structural damage evaluation can be obtained under whole loading conditions by single or several sensors only. No replacement or cleaning of sensor placements is necessary for that purpose. Furthermore, a noisy environment except structural vibrations, and so on, does not influence to the data acquisition system too much. Therefore, acoustic emission technique in structural health monitoring system is widely applied as a preferable nondestructive tool in various fields of industrial to biomedical engineering fields. 


\subsection{Onward features and prospective of present topic}

According to the strategy of the present writing, acoustic emission technique will be elaborated perspective to its historical criteria in structural health monitoring statistics. Following to its historical elongation, theoretical and experimental applications will be strategically approached. Afterward, some classical applications will be discussed before an elaborate discussion and conclusions of the technique to the structural health monitoring system.

\section{Fundamentals of AE technique to SHM applications}

\subsection{History of $\mathrm{AE}$}

Historically, the application of AE to structural health monitoring is not so new; however, the challenges of this monitoring strategy are still facing and progressing on time. Technologically, $\mathrm{AE}$ was investigated in the middle of twentieth century. In early stage, AE phenomena were realized in Germany by Forester [14] in transforming mechanical vibration into electrical voltage by electrodynamic transmitter-receiver system. They measured tiny voltage change due to resistance variations caused by martensite transformations in metallurgical experiments. However, AE experiments were officially founded by Kaiser [15] through the publication of his historical irreversibility theory, known as the "Kaiser effect" in 1950. The terminology "AE" was first in history published by Kaiser's pioneering work "Acoustic Emission" in 1961. Later on, Obert, Schofield, Drouillard, Yokomichi, Ikeda, Matsuoka, and Kishinoue did enormous efforts to apply AE in various fields until the end of twentieth century [16-20]. Thus, the application of AE became familiar as a well-known nondestructive tool to different fields in structural health monitoring applications.

\subsection{AE technique}

Acquisition of transient elastic wave generated due to the sudden change of material stress for any external stimulus locally or globally to the material generates acoustic emission waves. The generated waves propagate through the surface of the material, and therefore, acquisition of these elastic waves and conversion of these waves to electrical signals for visualization and analysis refer to the fundamentals of AE testing. In AE testing, a piezoelectric transducer, generally called as AE sensor, is placed on the surface of the material to be tested. The transducer responds to the dynamic motion generated by the elastic wave as mechanical motion and converts it to an electrical voltage signal, which is often called as AE signal. AE sensor is selected based on the operating frequency. Therefore, different types of AE sensors are commercially available based on their applicable frequencies and sensitivities [21-23].

A fundamental AE testing system consists of a sensor (AE sensor), a preamplifier, main amplifier with appropriate filters, and data acquisition system along with display (oscilloscopes, personal computer with data acquisition software, and data transferring devices like $\mathrm{AD}$ conversion system). As AE signals are very small, it is boosted by preamplifier to gain at low signal-to-noise ratio. Later on, AE signals are amplified again and passed through band 


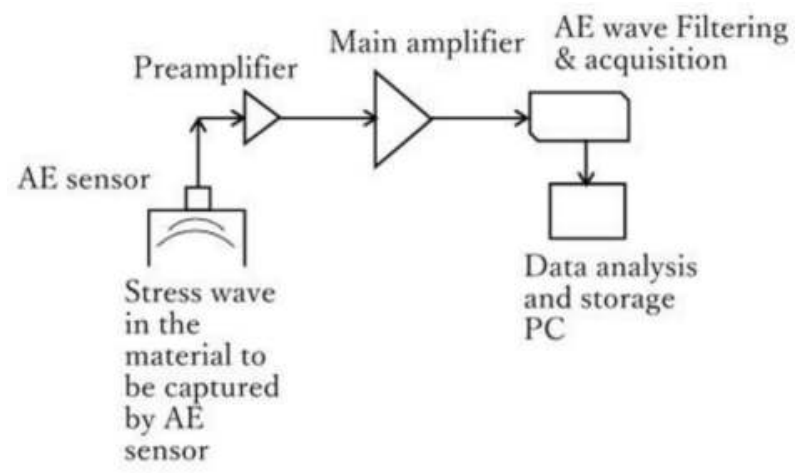

Figure 1. Fundamentals of AE technique.

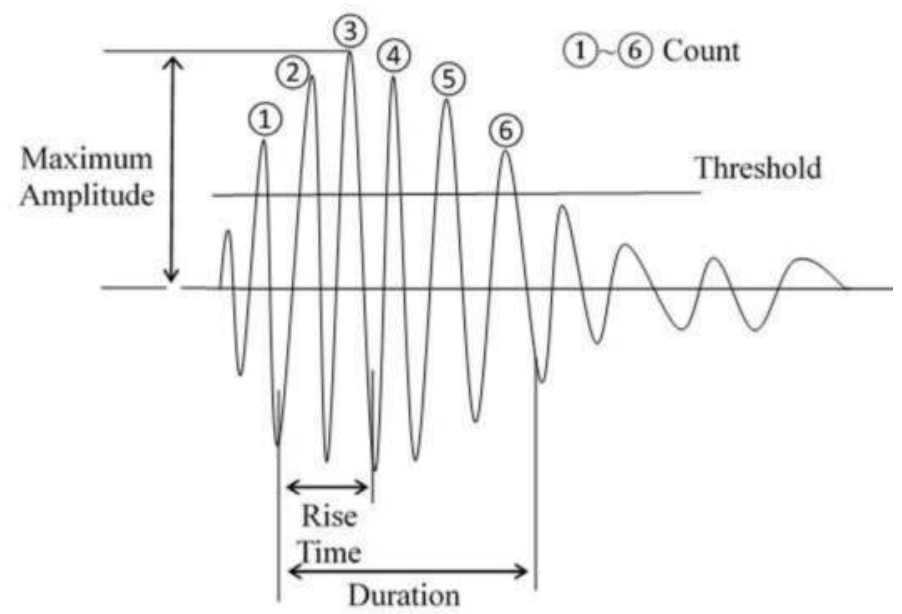

Figure 2. Common parametric features in an AE hit.

pass filters before storing to the mainframe of personal computer (PC) for analysis to any of desired features. The abovementioned AE technique is explained by the following schematic diagram as shown in Figure 1 [24-26].

\subsection{Important $\mathrm{AE}$ parametric features}

In AE testing, as shown in Figure 1, AE signals are received and visualized to the display of the acquisition device when AE sensor is attached to the material surface (object to be tested) by adhesives or tape and is excited by the generated stress wave at the material. AE waves are then saved to PC for further synthesis to characterize the damage inside the test object. Then, parametric features are widely used in analyzing or monitoring damage inside an object by AE NDT, as shown in Figure 2 [13, 27]. 
AE event: The time domain or frequency domain of acoustic emission signals represents parametric features due to the elastic wave generated inside the material. It is the total AE wave representation during the $\mathrm{AE}$ testing.

AE hit: It is represented as the AE signal from one channel and crosses the user-defined threshold. There can be multiple hits in an AE event or an AE test of multiple channels.

Maximum amplitude: Maximum amplitude (amplitude) is the greatest amplitude of an AE hit measured in voltage or in decibels $(\mathrm{dB})$. AE signals with maximum amplitude below the user-defined threshold line are not recorded as AE signals.

Counts: Counts are referred to the numbers of pulses of an AE hit, which cross the userdefined threshold value.

Rise time: Rise time is defined as the time span of an AE hit from its first threshold crossing amplitude to its maximum amplitude.

Duration: Duration is defined as the time span of an AE hit from its first threshold crossing amplitude to its last threshold crossing amplitude.

Energy: It is the area below the detection envelope within the duration of an AE hit.

Peak frequency: It is the frequency component $(\mathrm{kHz})$ corresponding to the maximum amplitude in an AE wave spectrum.

Average frequency: It is the average frequency in an AE hit. It is associated with duration and count and can be calculated from dividing "count" by "duration." It can roughly represent the signal frequency (when AE waveform is not possible to record). It represents complete acoustic emission impact signal.

Center frequency: It is the frequency component $(\mathrm{kHz})$ corresponding to the center of gravity in an AE wave spectrum.

Initial frequency: It indicates the initial condition of an AE spectrum. It is calculated from dividing "counts" until peak by "rise time."

Reverberation frequency: It is calculated from the relation derived from total count to initial count divided by the relation derived from duration to rise time.

RA value: It is calculated from rise time divided by maximum amplitude (amplitude). It is the reciprocal of gradient in AE signal waveform and represents the type of cracks in the unit of ms/V.

\subsection{AE sensors and data acquisition}

In a practical $\mathrm{AE}$ experiment, generally piezoelectric sensor is widely used as AE sensor. It is normally a contact-type sensor consisted of piezoelectric element protected by hard metal housing and connected by an electric connector for transmitting the generated electric effects. The sensing system is based on the piezoelectric effect out of lead zirconate titanate (PZT). This type of sensor is relatively cheap and highly sensitive and converts the mechanical movement to electrical voltage in AE experiments efficiently. 


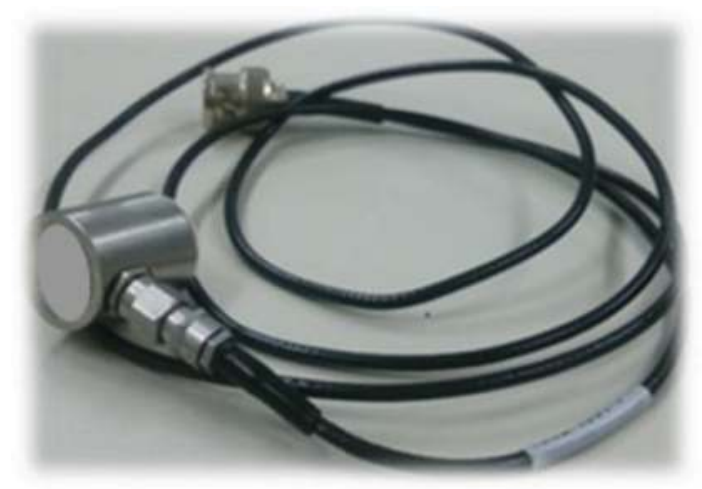

Figure 3. A typical AE sensor with connecting cable.

Selection of an appropriate AE sensor depends on the demanded frequency in the experiment. As the propagation of elastic wave is heavily affected by the property of the propagation path and the propagation mechanism to the $\mathrm{AE}$ sensor, the frequency content of the propagating elastic wave plays a very important role in the selection of the suitable AE sensor in AE tests (Figure 3).

Appropriate sensors for AE testing to pressure vessels, storage tanks, heat exchangers, piping, reactors, aerial lift devices, nuclear power plants, and biomedical fields are well prepared based on their required frequency range. In general, based on the frequency range for AE tests, sensors are classified accordingly as low-frequency range sensor $(20-100 \mathrm{kHz})$, middle or standard range sensor (100-400 kHz), and high-frequency range sensor ( $400 \mathrm{kHz})$. Different companies such as Physical Acoustic Corporation (PAC), Vallen Systeme Company, and so on produce their sensors regarding the type of commercial AE sensor with high sensitivity $[25,28-30]$.

\section{AE source location}

Source location plays a significant role in AE technique. It is an advantageous facility in AE technique when compared with many other NDTs. It makes the characterizing of damage propagation behavior and the overall damage monitoring system well understandable and well predictable.

It is already mentioned that many $\mathrm{AE}$ hits can be taken in one $\mathrm{AE}$ event by placing many $\mathrm{AE}$ sensors in AE testing. One channel (one AE sensor) can record one AE hit, and thus, multiple channels (multiple AE sensors) can record multiple AE hits. When AE sensors place at different places at a suitable sensor to sensor distance (sometimes carefulness in signal wavelength is necessary) according to the desired inspection area, all AE hits are recorded by the system with different signal traveling times based on the different distances of sensors to the signal source. This traveling time is termed as "arrival time" in AE source location. Knowing the traveling time or arrival time of each hit from the signal acquisition system and multiplying it with the AE signal traveling velocity in that material, signal traveling distance from the 
source to each AE sensor is calculated. For simplicity, wave velocity for a particular material is assumed constant in general AE source location technique. However, considering different geometric or traveling effects, like wave reflection due to material inhomogeneity, various wave modes ( $p$-mode, s-mode, etc.) precise velocity calculation is necessary for improving the accuracy of AE source location technique. Based on the number of AE sensors connected to the acquisition system, several source location techniques have been developed already. Several AE source location techniques are discussed later.

\subsection{Linear source location technique}

A fundamental and very commonly used technique is the linear type of AE source location technique. At least two AE sensors (minimum required number of AE sensors in AE source location technique) are necessary in this technique. Linear type of structures such as bridge and pipe is used for measurements by this technique. This technique is very simple and easy to apply. It is also called as one degree AE source location technique [13].

In linear source location technique, two sensors are placed to an appropriate distance, and therefore, the time of arrivals from two sensors is collected. Based on the difference in signal arrival time, source location is defined. For example, if the source location is located at the middle point of two sensors, the difference between two arrival times is zero. Otherwise, the arrival time will be different. It is considered in this technique that shorter arrival time is the closer source to the receiving sensor. Thus, the source length is calculated by multiplying the arrival time with wave traveling velocity. The schematic of a linear source location technique is shown in Figure 4, where AE source is mentioned by s. Similarly, $s_{1}, s_{2}$ and $t_{1}, t_{2}$ indicate as the $\mathrm{AE}$ sensor 1, AE sensor 2 and time of arrival to sensor 1, time of arrival to sensor 2 , respectively. Furthermore, $l, l_{2}$, and $l_{1}$ indicate the distance between two sensors $\left(\mathrm{s}_{1}, \mathrm{~s}_{2}\right)$, axial distance of AE source to sensor 1, and axial distance between AE source and the midpoint $(l / 2)$ of two AE sensors, respectively.

The mathematical relations of the linear source location algorithm are explained as the following equations, where $\Delta t$ indicates the time difference between two arrival times to sensor 1 and sensor 2 and $v$ indicates the AE wave velocity [31].

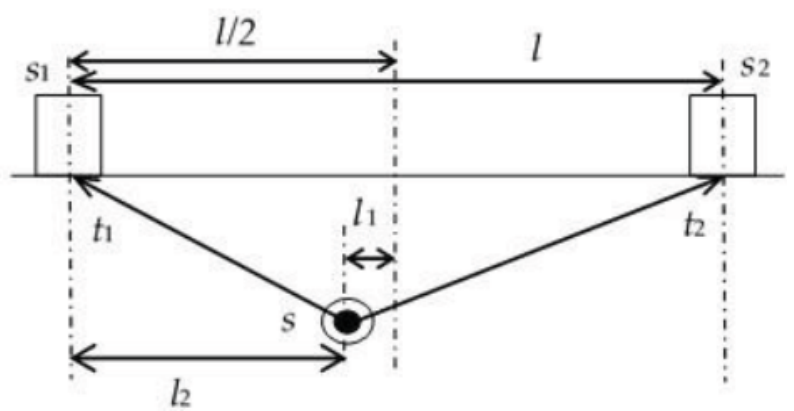

Figure 4. Schematic of linear source location technique. 


$$
\begin{aligned}
& l_{1}=\frac{1}{2}\left(t_{1}-t_{2}\right) \cdot v=\frac{1}{2} \Delta t \cdot v \\
& l_{2}=\frac{1}{2} l-l_{1}=\frac{1}{2}(l-\Delta t \cdot v)
\end{aligned}
$$

\subsection{Two-dimensional source location technique}

Planner or two-dimensional source location technique requires three or more AE sensors to be placed on a plane for identifying AE source. Three sensors generate three hyperbolae, which intersect each other on the monitoring plane at a common interceding point or cross-sectional point, and the point is termed as the AE source. Theoretically, three sensors are sufficient for identifying the source in two-dimensional technique; however, another extra sensor, which is called as reference sensor, improves the accuracy of the source location technique. Therefore, the placement of four sensors in a rectangular sensor array generates six sensor pairs. Calculating time of arrival from each sensor pair and correlating according to the following relations planner or two-dimensional AE source location are done.

The simple algorithm for calculating two-dimensional AE source location is based on the following relations [32].

$$
\begin{gathered}
x_{s}{ }^{2}+y_{s}{ }^{2}=r_{s}{ }^{2} \\
(\Delta x)_{i}{ }^{2}+(\Delta y)_{i}{ }^{2}=r_{i}{ }^{2} \\
(\Delta x)_{j}{ }^{2}+(\Delta y)_{j}{ }^{2}=r_{j}{ }^{2}
\end{gathered}
$$

where $\Delta x=x_{s}-x$ and $\Delta y=y_{s}-y$, when $x$ and $y$ indicate the coordinates of sensor positions; however, for the source indication, the suffix $s$ is used. However, $s_{0}$ indicates the reference sensor. Furthermore, suffixes $i$ and $j$ indicate the general number of positioning and measuring sensors, respectively, for example, in Figure 5, $s_{i}$ indicates for sensor 1, and $s_{j}$ indicates for sensor 2. Similarly, $r_{i}, r_{j^{\prime}}$ and $r_{s}$ indicate the distances from the corresponding sensors to source. Applying simple solutions of above Eqs. (3)-(5), the source distance, $r_{s}$ can be calculated as follows:

$$
r_{s}=\frac{1}{2}\left[\frac{U_{i}}{x_{i} \cos \theta+y_{i} \sin \theta+d_{i}}\right]=\frac{1}{2}\left[\frac{U_{j}}{x_{j} \cos \theta+y_{j} \sin \theta+d_{j}}\right]
$$

In Eq. (6), $U_{\mathrm{i}^{\prime}}, U_{\mathrm{j}^{\prime}}, d_{\mathrm{i}^{\prime}}$ and $d_{\mathrm{j}}$ are defined as follow:

$$
\begin{gathered}
U_{i}=x_{i}^{2}+y_{i}^{2}-d_{i}^{2} \\
U_{j}=x_{j}^{2}+y_{j}^{2}-d_{j}^{2} \\
d_{i}=r_{i}-r_{s}=v \cdot \Delta t_{i 0} \\
d_{j}=r_{j}-r_{s}=v \cdot \Delta t_{j o}
\end{gathered}
$$




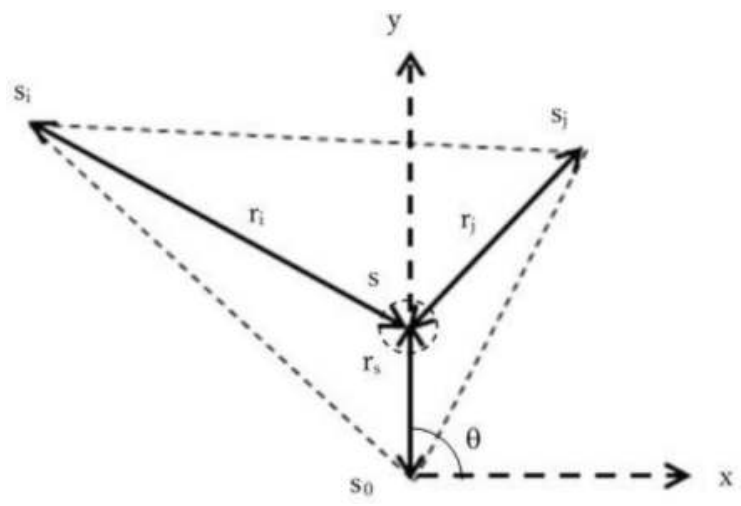

Figure 5. Geometrical representation of two-dimensional source location algorithm.

Similarly, the source angle $(\theta)$ can be calculated as follows:

$$
\theta=\phi+\psi
$$

In Eq. (11), the parameters $\phi$ and $\psi$ can be calculated by rearranging Eq. (6), and thus, the two-dimensional source location can be found.

Similarly, three-dimensional source location is also possible to calculate in AE source location technique by increasing the number of sensors. Many other modern source location techniques are available $[10,13]$ and are still introducing to AE fields by AE researchers from all over the world.

\section{Smart applications of AE technique}

The application of acoustic emission for fault detection or condition monitoring in structural health monitoring (SHM) field is versatile. Since it is a noninvasive technique, it is widely applied in different fields of nondestructive testing (NDT), nondestructive evaluation (NDE), and nondestructive monitoring (NDM) for many engineering applications. Similar application of AE in seismology is well known from its beginning of implementations. Further, recently, applications of $\mathrm{AE}$ in biomedical engineering field have attracted many scientists and engineers for its smart applications in condition monitoring as well. Several examples of $\mathrm{AE}$ applications are mentioned later.

\subsection{Industrial applications in SHM}

Out of many industrial applications of AE technique, several are mentioned as monitoring of pressure vessel, storage tank, structural materials, composites, concrete structure, steel structure, bridge, aircraft, gear, ceramics, ceramic components, and so on. In maximum cases of inspection, 
standard AE features are evaluated experimentally under satisfactory experimental environments to practical application and correlated with standard values. For example, pressure vessel or other high-pressure tanks are evaluated under cyclic loading pressure [8] up to lifetime number of loading. AE sensors are attached to the tank to be tested and connected to the data acquisition components such as preamplifier and filters, and finally, the digital acquisition system saves the data to the computer where the required AE parameters are evaluated for crack or damage evaluation. Multiple sensors are placed for evaluating the damage source as well. Pipe line or drill pipe fatigue damage is also evaluated frequently by AE technique. Although fatigue loading is a complex mechanism in operation, however, it is defined by cycle number in all fatigue tests, and therefore, fatigue damage is normally evaluated based on crack propagation under certain range of applied cyclic loads. Based on application criteria, drill pipe is subjected to cyclic stress in tension, compression, torsion, and bending. Bending and rotation produce alternation between states of loading at a localized point, which is most concern to damage under fatigue loading. In drilling test rig monitoring, frequent fatigue failures are tested based on critical rotary speed, maximum tension load, notch fatigue load, etc. [33].

Most bridges are tested based on their welded joints and connections under the combinations of loading and loading environments. The general frequency of monitoring bridges is 2 years. Under visual inspection, the necessity of shutting down of its weight capacity is done when damage is found. However, in AE inspection, lane closer is not necessary as it monitors data continuously for real-time forecasting of any damage. Therefore, bridge monitoring by AE technique has increased as well [34].

Material testing by AE technique is also widely applicable. Structural materials, ductile materials, brittle materials, and many other materials including composite materials are tested to evaluate their cracking, breaking, and damaging characteristics for different industrial and biomedical applications. Furthermore, microstructural studies including metallurgical characteristics of many materials are also tested by different smart AE tests.

Different aerospace structures are monitored by AE technique as well. Many sensors are possible to attach to different parts of aerospace structures easily, and therefore, damage monitoring even in minute level and damage location in multidimensional features are performed by $\mathrm{AE}$ technique. In many smart $\mathrm{AE}$ techniques, real-time wireless monitoring is done by applying wireless AE sensing systems. For example, NASA installed AE sensor-based alert system on the inside of the space shuttle Discovery's wing structure for avoiding the damage of its leading edge during the reentry to the Earth atmosphere [35].

\subsubsection{AE parametric analysis}

A material test result was conducted for showing the parametric analysis of AE technique [36]. The experiment was conducted for a cast iron specimen (ferrite) under tensile loading in an autograph tensile machine. Four AE sensors (R15 $\alpha$, Physical acoustics Ltd.), placed to the specimen for getting $\mathrm{AE}$ data due to crack damage, were connected to four preamplifiers and to a main amplifier with a gain of $40 \mathrm{~dB}$. AE data were collected by a digital oscilloscope, and the collected data were analyzed for the characterization inside a personal computer. For avoiding noise, appropriate threshold values were used. All the recorded AE hits were saved, 


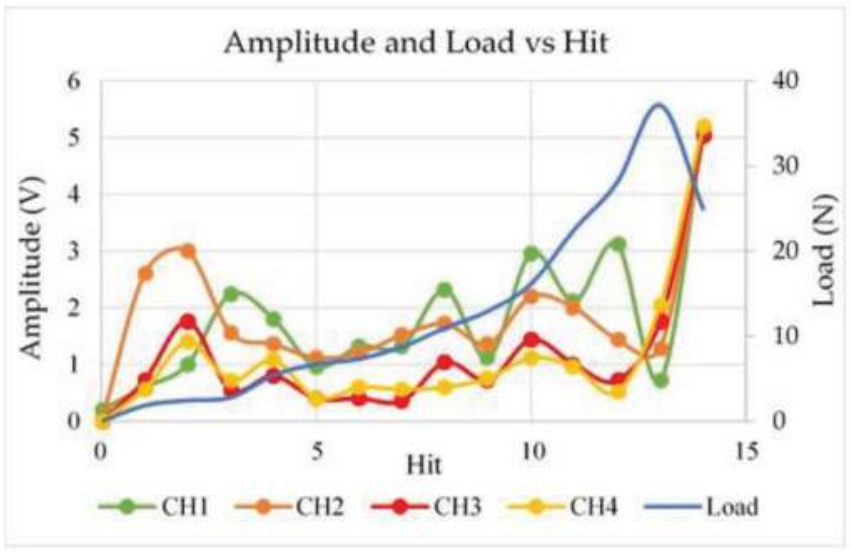

(a)

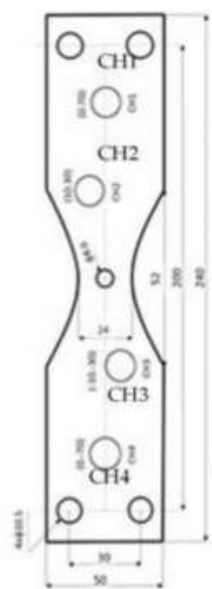

(b)

Figure 6. (a) Graphical representation of AE parametric distribution and (b) AE sensor positions in the specimen.

and $\mathrm{AE}$ parametric features were calculated to represent the characteristics of crack propagation in a ferrite material under tensile loads. Applied tensile loads were simultaneously saved to the computer for evaluation as well. One experimental result showing AE parametric distribution (amplitude distribution versus tensile loads compared to AE hits) is shown in Figure 6. In this experiment, according to the sensor positions, oscilloscope channels ( $\mathrm{CH}$ in figure) were defined. Furthermore, sensors 2 and 3 were placed near to the crack initiation position; sensor 1 was placed near to the loading chuck; sensor 4 was placed near to the specimen supporting chuck. Accordingly, sensor $2(\mathrm{CH} 2)$ and sensor $3(\mathrm{CH} 3)$ represented the maximum AE excited values in signal amplitude, whereas sensor 1 (CH1) showed the AE amplitude along with noise contamination from the loading chuck. Similarly, sensor $4(\mathrm{CH} 4)$ represented the minimum value as it was the farthest from the cracking position and far from loading nose. Furthermore, amplitude excitation and distribution values of $\mathrm{CH} 2$ and $\mathrm{CH} 3$ showed that at the initiation of cracking, amplitude excitations were high due to the tensile cracking at its early stage of AE hits, and after that, as cracking took the shear loading, the amplitude values went down until it gained the maximum values at the fracture stage at their end stage of AE hits.

\subsubsection{AE source location}

An experiment of two-dimensional source location technique as explained in Section 3.2 was conducted on a steel plate with generated artificial AE source based on Hsu-Nielsen [31, 37] technique. The arrival time was calculated according to the first signal recognition in AE hit as shown in Figure 7. Three experiments were conducted with three sensor distances, where sensorto-sensor distance was kept constant among three sensors in each experiment. Accordingly, the source location represented by source distance $\left(r_{s}\right)$ and source angle $(\theta)$, as shown in Figure 4, was calculated. The results were compared with its actual measured data (known before for comparison) and found good agreement as well. The results are summarized in Table 1. 


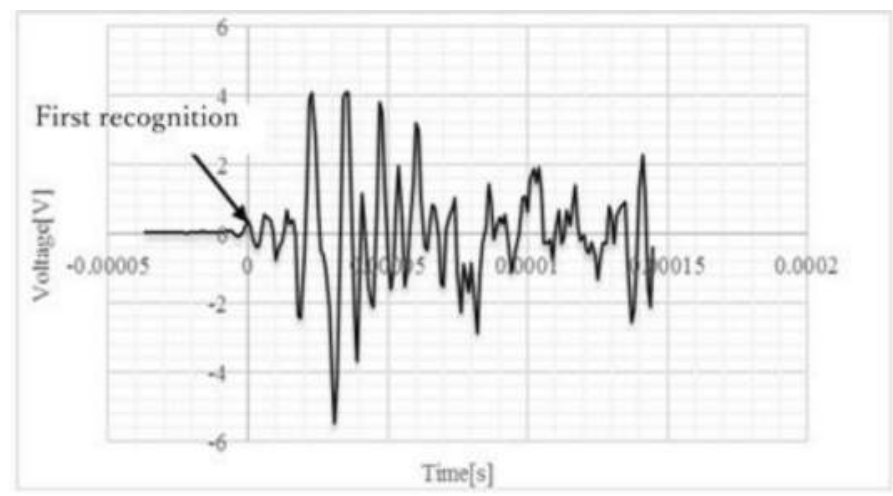

Figure 7. AE hit showing its initial recognition position of arrival signal.

\begin{tabular}{lllllll}
\hline Distance & Actual, $\boldsymbol{\theta}$ & Experimental, $\boldsymbol{\theta}^{\prime}$ & Actual, $\mathbf{r}_{\mathrm{s}}$ & Experimental, $\mathbf{r}_{\mathrm{s}}$ & Error of $\mathbf{r}_{\mathrm{s}}(\%)$ & Error of $\boldsymbol{\theta}(\%)$ \\
\hline 200 & 90 & 89.42 & 0.1414 & 0.1424 & 0.69 & 0.64 \\
250 & 90 & 89.67 & 0.1768 & 0.1775 & 0.41 & 0.37 \\
300 & 90 & 89.68 & 0.2121 & 0.2130 & 0.41 & 0.36 \\
\hline
\end{tabular}

Table 1. Results of AE two-dimensional source location.

\subsection{AE applications in biomedical health monitoring}

A successful history of AE technique in fault detection and condition monitoring encourages research to apply it to biomedical engineering field as well. Since AE technique perfectly evaluates and monitors any discontinuity and internal damage of a structure, it is successfully applied in detecting the integrity condition of human bones and joints, particularly knee joints. As detailed functional assessment of knee joint includes to identify any irregularity among its internal anatomical structures, AE sensors are installed to the knee joint and internal damages are evaluated. A common knee disease, particularly of elderly people, is osteoarthritis. It causes due to the damage of internal cartilage of knee joint. This disease causes the disability of people, and therefore, its prevalence is predicted to increase as a result of aging people in an aging society. The damage of cartilage brings the raw bone-end in contact and causes several knee diseases. In the worst case of osteoarthritis, operation is needed to replace by artificial joint with mixed satisfaction of the patient. Application of AE technique in diagnosis of cartilage damage is interestingly applied as well.

Accordingly, experiments of AE technique for identifying the integrity of knee joint were conducted [38]. Four AE sensors were placed to the knee joint, and AE data (AE features) were collected as shown in Figure 8. AE parameters from the knee were collected under the dynamic loading condition of knee joint by several stand-sit-stand motions of participants. Both 


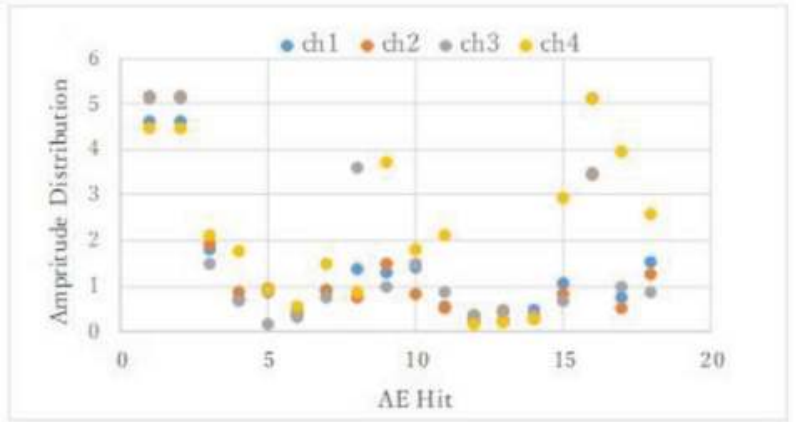

(a)

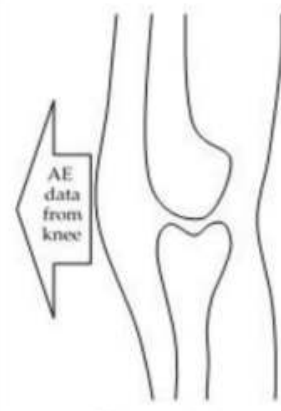

Knee joint

(b)

Figure 8. (a) Sample result of AE amplitude distribution for four sensors from the knee joint experiments and (b) schematic of knee joint from AE sensor data were collected.

healthy participants and patients of knee osteoarthritic disease joined to the experiments. A sample result of AE amplitude distribution compared with AE hit is presented in Figure 7. Acquisition of AE data is perfectly specified in the figure. All channels received sufficient AE data for identifying the internal conditions of knee joint. Thus, experimental results showed that monitoring of knee condition is possible by applying the AE technique successfully to knee joint and to others.

\section{Conclusions}

A feature outlook of acoustic emission (AE) technique related to the structural health monitoring has been presented in this chapter. A brief history and chronology of AE monitoring are discussed with adequate references. Basic understandings about $\mathrm{AE}$ technique, its experimental methodology, and applications are also summarized in this chapter. A list of frequently used AE parameters is also added here. Useful definitions of AE parameters are provided in this parametric list for understanding the theme of AE parametric analysis. Almost all of the structural health monitoring based on AE technique are conducted by the mentioned parameters. Three examples covering three important applications of $\mathrm{AE}$ technique are summarized at the end of the chapter. Two major wings of $\mathrm{AE}$ technique in structural health monitoring are $\mathrm{AE}$ parametric analysis and $\mathrm{AE}$ source location. Both of these applications are explained with appropriate examples along with experimental results in material damage and crack propagations. A smart application of AE technique in biomedical engineering field is also mentioned in this chapter as a promising scope of AE technique for the future versatile solutions. Extension of this work can be found in the future publications as well. Thus, the chapter has made easier for wider and fruitful understanding of $\mathrm{AE}$ technique in structural health monitoring to all of its readers. 


\section{Acknowledgements}

The author expresses his sincere thanks and gratitude to all funding organizations including MEXT, Collaborating Company, Daishin Co. Ltd., and Saga University as well as to all graduate and undergraduate students of his laboratory for their continuous supports to continue all of his research topics. The author is particularly indebted to his biomedical research partner, Dr. Suya Ide (M.D.), for his enormous supports and guidance in continuing the research. The author is also obliged to all of his friends and colleagues, particularly to all participants of his research for having successful goal.

\section{Author details}

Md. Tawhidul Islam Khan

Address all correspondence to: khan@me.saga-u.ac.jp

Department of Mechanical Engineering, Saga University, Saga, Japan

\section{References}

[1] Miller RK, McIntire P, editors. Acoustic Emission Testing. Nondestructive Testing Handbook. 2nd ed. Vol. 5. American Society for Nondestructive Testing. p. 603

[2] Ono K. Trends of recent acoustic emission literature. Journal of Acoustic Emission. 1993;12:177-198

[3] Sachse W, Kim KY. Quantitative acoustic emission and failure mechanics of composite materials. Ultrasonics. 1987;25:195-203

[4] Meyendorf N, Frankenstein B, Schubert L. Structural health monitoring for aircraft, ground transportation vehicles, wind turbine and pipes-prognosis. In: Proceedings of 18th World Conference on Nondestructive Testing; 16-20 April 2012; Durban, South Africa

[5] Holroyd TJ, Randall N. Use of acoustic emission for machine condition monitoring. British Journal of Non-Destructive Testing. 1993;35:75-78

[6] Gorman M, Prosser WH. Application of normal mode expansion to acoustic emission waves in finite plates. Journal of Applied Mechanics. 1996;63:555-557

[7] Hamstad MA, Ogallagher A, Gary J. A wavelet transform applied to acoustic emission signals: Part 2: Source location. Journal of Acoustic Emission. 2002;20:62-82

[8] Laksimi A, Herve C, Ennaceur C, Cherfaoui M. AE Study of damage evolution in pressure vessels under cyclic loading. In: Proceedings, 30th European Conference on 
Acoustic Emission Testing \& 7th International Conference on Acoustic Emission; 12-15 September 2012; Granada, Spain

[9] Gorman MR, Prosser WH. AE source orientation by plate wave analysis. Journal of Acoustic Emission. 1991;9:283-288

[10] Christian UG, Ohtsu M, editors. Acoustic Emission Testing. Springer; 2008. DOI: 10.1007/ 978-3-540-69972-9

[11] Rindorf HJ. Acoustic Emission Source Location in Theory and in Practice. Vol. 2. Bruel and Kjaer Technical Review; 1981. pp. 3-44

[12] Kaita I, Enoki M. Acquisition and analysis of continuous acoustic emission waveform for classification of damage sources in ceramic fiber mat. Materials Transactions. 2007;48:1221-1226

[13] https://www.nde-ed.org/EducationResources/CommunityCollege/Other\%20Methods/ AE/AE_Intro.htm [Accessed: May 22, 2018]

[14] Forster F, Scheil E. Akustische Untersuchung der Bildung von Martensitnadeln (acoustic study of the formation of matensile needels). Zeitschrift für Metallkunde. 1936;28:245-247

[15] Kaiser J. A study of acoustic phenomena in tensile tests. [Dr.-Ing. dissertation]. Technical University of Munich; 1950

[16] Obert L, Duvall W. The microseismic method of predicting rock failure in underground mining, Part II: Laboratory experiments. Report of Investigations 3803. Washington D.C.: U. S. Bureau of Mines. 1945

[17] Obert L. The microseismic method: Discovery and early history. In: Hardy Jr, Leighton FW, editors. Proceedings 1st Conference on AE/MS. Geologic Structures and Materials. Clausthal, Germany: Trans Tech Publication; 1977

[18] Schofield BH. Acoustic emission under applied stress. Report ARL-150. Boston: Lessels and Associates; 1961

[19] Kishinoue F. An experiment on the progression of fracture (a preliminary report). Jishin 6:24-31 (1934) translated and published by Ono K. Journal of Acoustic Emission. 1990;9:177-180

[20] Yokomichi H, Ikeda I, Matsuoka K. Elastic wave propagation due to cracking of concrete. Cement Concrete Japan. 1964;212:2-6

[21] Ohtsu M, Ono K. A generalized theory of acoustic emission and Green's functions in a half space. Journal of Acoustic Emission. 1984;3:124-133

[22] Beatttie AG. Acoustic emission, principles and instrumentation. Journal of Acoustic Emission. 1983;2:95-128

[23] Nishinoiri S, Enoki M. Development of in-situ monitoring system for sintering of ceramics using laser AE technique. Progress in AE XII:69-76, JSNDI; 2004 
[24] Ohtsu M. Acoustic emission characteristics in concrete and diagnostic applications. Journal of Acoustic Emission. 1987;6:99-108

[25] MISTRAS Group Inc. Express-8 AE System user's Manual, Rev 0, Part\#: 7050-1000. Physical Acoustic Corporation; 2014

[26] Khan M, Islam T, Nagao T, Kondo Y, Teramoto K, Hattori N. Monitoring the fatigue damage in ductile cast iron by $\mathrm{AE}$ technique. International Journal of COMADEM. 2015;18:27-33

[27] Shiotani T, Nakanishi Y, Iwaki K, Luo X, Haya H. Evaluation of reinforcement in damaged railway concrete piers by means of acoustic emission. Journal of Acoustic Emission. 2006;23:260-271

[28] Hatano H, Watanabe T. Reciprocity calibration of acoustic emission transducers in Rayleigh wave and longitudinal wave sound field. The Journal of the Acoustical Society of America. 1997;101:1450-1455

[29] Hsu NN, Breckenridge FR. Characterization and calibration of acoustic emission sensors. Materials Evaluation. 1981;39:60-68

[30] Vallen Systeme GmbH. Acoustic Emission Sensors Specification; 2015. Available from: http://www.vallen.de/zdownload/pdf/sov1507.pdf [Accessed 2018-07-03]

[31] Khan M, Islam T, Nagafuchi S, Hassan M. Structural damage localization by linear technique of acoustic emission. Open Journal of Fluid Dynamics. 2014;4:425-432

[32] Khan TI, Hassan M, Takata R. Effect of wave velocity in two-dimensional AE damage locationon a steel plate. International Journal of COMADEM. 2017;20:1-5

[33] Gomera VP, Sokolov VL, Fedorov VP, Okotnikov AA, Saykova MS. Inspection of the pressure vessel used in petrochemical with AE examination. In: Proceedings, EWGAE2010; 8-10 September 2010; Vienna

[34] Shiotani T, Aggelis DG, Makishima O. Global monitoring of concrete bridge using acoustic emission. The Journal of the Acoustical Society of America. 2007;25:308-315

[35] Chlada M, Prevorovsky Z. Remote AE monitoring of fatigue crack growth in complex aircraft structures. In: Proceedings, 30th European Conference on Acoustic Emission Testing \& 7th International Conference on Acoustic Emission; 12-15 September 2012; Granada, Spain

[36] Rashid AA, Khan Md TI, Hidaka R. Analysis of acoustic emission and crack propagation in ductile cast iron. In: Proceedings, 21th Acoustic Emission Symposium; 9-10 November 2017; Tokushima, Japan

[37] Mostofapour A, Davoodi S. A method for acoustic source location in plate-type structure. Mechanical Systems and Signal Processing. 2017;93:92-103

[38] Islam KT, Harino Y. Integrity analysis of knee joint by acoustic emission technique. Journal of Multimodal User Interfaces. 2015;10:319-324 